\title{
Unaltered respiratory-related evoked potentials after acute diaphragm dysfunction in humans
}

\author{
M. Bezzi*,+, C. Donzel-Raynaud*, , C. Straus ${ }^{\#,}$, C. Tantucci ${ }^{+}$, M. Zelter $^{\#, \uparrow}$, J-P. Derenne*,ף, \\ T. Similowski*,
}

Unaltered respiratory-related evoked potentials after acute diaphragm dysfunction in humans. M. Bezzi, C. Donzel-Raynaud, C. Straus, C. Tantucci, M. Zelter, J-P. Derenne, T. Similowski. (C) ERS Journals Ltd 2003.

ABSTRACT: Respiratory muscles play an important role in the origin of respiratory sensations. Data dissecting the role of the diaphragm and other inspiratory muscles are scarce. This study aimed to determine the impact of diaphragm dysfunction following inspiratory resistive loading on respiratory-related evoked potentials considered as a neurophysiological substrate of certain types of respiratory sensations.

Altogether, nine subjects aged 25-50 yrs (six females) participated in the study. Transdiaphragmatic pressure output of cervical magnetic stimulation (with subdivision in oesophageal and gastric component), and respiratory-related evoked potentials (C3 and $\mathrm{C4}$ derivations in the international 10-20 system) following mid-inspiratory occlusions were studied before and after an inspiratory-resistive loading challenge.

Predominant diaphragm dysfunction was observed in seven subjects (average $28 \%$ reduction in transdiaphragmatic pressure, from $27.25-19.91 \mathbf{c m H}_{2} \mathbf{0}$, with increased oesophageal-to-gastric pressure ratio). The latencies and amplitudes of all the components of the respiratory-related evoked potentials were unchanged.

The study concluded that predominant diaphragm fatigue does not affect respiratoryrelated evoked potentials.

Eur Respir J 2003; 22: 625-630.

Respiratory sensations are commonly considered to result from the balance between the central ventilatory drive, the corresponding production of force by ventilatory muscles, and a complex afferent feedback [1]. The neural processing of changes associated with inspiratory loads allows normal humans to detect them, identify their nature (e.g. resistive versus elastic), estimate their magnitude, and in turn to adapt to their presence. The corresponding sensations provide an alarm system of which the deficiency can be a relevant pathophysiological determinant of respiratory diseases (e.g. certain severe forms of asthma are associated with a blunted perception of inspiratory loads [2]).

There is considerable evidence documenting the role of inspiratory muscles and the rib cage in the detection of added inspiratory loads [3]. Respiratory muscle function also modulates respiratory sensations. Inspiratory strength training does not alter load detection but reduces the perceived magnitude of both resistive and elastic loads [4]. Conversely, globally weakening inspiratory muscle exacerbates the sense of inspiratory efforts $[5,6]$. This is similar to that occurring in upper limb muscles where the development of fatigue increases the perceived heaviness of a lifted object [7], and
*Laboratoire de Physiopathologie Respiratoire, Service de Pneumologie, and ${ }^{\#}$ Service Central d'Explorations Fonctionnelles Respiratoires, Groupe Hospitalier Pitié-Salpêtrière, Assistance Publique - Hôpitaux de Paris, 'Unité Propre de Recherche de l'Enseignement Supérieur EA 2397, Université Pierre et Marie Curie Paris VI, Paris, France and ${ }^{+}$Servizio di Medicina Interna 1, Spedali Civili, and Universita di Brescia, Brescia, Italy.

Correspondence: T. Similowski, Laboratoire de Physiopathologie Respiratoire, Service de Pneumologie et de Réanimation, Groupe Hospitalier Pitié-Salpêtriêre, 47-83, Bd de 1'Hôpital, 75651 Paris Cedex 13, France.

Fax: 33142176708

E-mail: thomas.similowski@psl.ap-hop-paris.fr

Keywords: Diaphragm, dyspnoea, fatigue, respiratory muscles, respiratory-related evoked potentials, respiratory sensation

Received: March 42003

Accepted after revision: June 162003

This study was supported by Contrat de recherche triennal "Legs Poix" de la Chancellerie des Universités de Paris, and Association pour le Développement et l'Organisation de la Recherche en Pneumologie (ADOREP), Paris, France. illustrates the role of respiratory muscle afferents in the genesis of respiratory sensations. Another illustration of this contribution is provided by the neurophysiological evidence of a cortical processing of respiratory muscle afferents. The stimulation of intercostal [8] and phrenic [9, 10] afferents indeed evoke cerebral potentials in humans. Coherently, diaphragm fatigue has been shown to alter phrenic cortical potentials in the cat [11]. However, in man such changes do not seem to have been reported.

A simple means to study the cortical processing of respiratory afferents is to record the brain activity following repeated inspiratory occlusions at the airway opening (respiratory-related evoked potentials (RREP)), even though the exact source of this activity is not precisely known. The amplitude of the first positive component of the RREP varies with the intensity of the inspiratory effort required to overcome a resistive inspiratory load [12]. It is related to both the corresponding transdiaphragmatic pressure and subjective magnitude estimation [12, 13]. It is thus possible that respiratory muscle afferents participate in the genesis of the RREP. In this case, observing changes in the amplitude of the somatosensory component of the RREP in response to 
acute inspiratory muscle dysfunction would substantiate the corresponding changes in the sensory processing of inspiratory muscle information.

The contribution of the diaphragm and of extradiaphragmatic inspiratory muscles to respiratory sensations appears to be different $[14,15]$. The present study aimed at examining the effects of experimentally reducing the pressure generation ability of the diaphragm, while sparing rib cage muscle as much as possible [16], on the characteristics of RREP.

\section{Materials and methods}

\section{Subjects}

After completion of the French legal procedure for biomedical research in human volunteers, nine White subjects participated in the study (six females, three males; aged 25-50 yrs; height 159-192 cm; weight 48-100 kg). They were informed in detail of the purpose of the study and methods used, and gave written consent. The subjects were instructed to avoid sleep deprivation during the $48 \mathrm{~h}$ preceding the experiments, to refrain from any consumption of alcohol and psychotropic substances, and to eat lightly on the day of the study.

\section{Measurements and procedures}

Breathing circuit. The subjects, wearing a noseclip, breathed room air through a flanged mouthpiece and a heated HansRudolph pneumotachograph (3700 series; Hans Rudolph, Kansas City, MO, USA) connected to a $\pm 2 \mathrm{cmH}_{2} \mathrm{O}$ linear differential pressure transducer (Validyne, Northridge, CA, USA) in order to measure the ventilatory flow. The pneumotachograph was assembled in series to a nonrebreathing twoway valve (2600 series; Hans Rudolph) of which the inspiratory port could be occluded by an inflatable balloon (Hans-Rudolf 9340 series occlusion valve and 9330 series compressor; Hans Rudolph). Mouth pressure was measured from a side port of the mouthpiece, using a differential pressure transducer (DP 15-32; Validyne).

Abdominal displacements. Changes in abdominal circumference were monitored with a mechanical strain gauge (Nihon Kohden, Tokyo, Japan) attached to an elastic belt placed at the level of the umbilicus.

Pressures. Oesophageal pressure $(P$ es $)$ and gastric pressure $(P$ ga) were measured with two balloon-tipped catheters (thinwalled balloon sealed over a polyethylene catheter with distal side holes, $60 \mathrm{~cm}$ length, $1.7 \mathrm{~mm}$ inside diameter) connected to two Validyne MP45 linear differential pressure transducers (Validyne, Northridge, CA, USA). Transdiaphragmatic pressure $(P$ di) was obtained on-line by connecting the $P$ es and $P$ ga catheters to a third transducer of the same type. $P$ di was continuously displayed to the subjects on a computer screen.

Surface diaphragm electromyograms. Surface recordings of the right and left costal diaphragmatic electromyographical activity were obtained using disposable silver cup electrodes taped to the skin [17], in order to assess the validity and reproducibility of phrenic nerve stimulation [18]. The signals were fed to amplifiers (Nihon Kohden), with a $10 \mathrm{kHz}$ sampling rate and a $20 \mathrm{~Hz}-5 \mathrm{kHz}$ bandwidth.

Cervical magnetic stimulation. Cervical magnetic stimulation (CMS) was carried out according to the previously described technique [19, 20], using a Magstim 200 stimulator (Magstim, Whitland, Dyfed, UK) equipped with a $90 \mathrm{~mm}$ circular coil (peak magnetic field 2.5 Teslas). All the stimulations were delivered at relaxed end-expiration as judged from the abdominal circumference trace, as well as the $P$ es and $P$ di traces, with the glottis closed. The maximal stimulation intensity of the stimulator was systematically used, after verifying from a simplified recruitment curve (amplitude of diaphragm quasi-synchronised action potentials versus stimulation intensity) that it corresponded to a supramaximal response. Diaphragm twitches were retained for analysis only if the amplitude of the corresponding electromyographic responses to CMS matched the previously determined maximum amplitude, before and after fatigue. The amplitude of the oesophageal, gastric, and transdiaphragmatic pressures swings produced by the CMSinduced diaphragm twitches ( $P$ es,tw, $P$ ga,tw, and $P$ di,tw, respectively) were measured from baseline to peak.

Respiratory-related evoked potentials. The electroencephalographic activity (EEG) was recorded using standard surface electrodes placed at the scalp positions $\mathrm{C}_{\mathrm{Z}}, \mathrm{C}_{3}, \mathrm{C}_{4}$ on the basis of the international 10-20 system [21]. $C_{3}$ and $C_{4}$ were referenced to $\mathrm{C}_{Z}$ to record the left and right activity, respectively. The ground electrode was placed on the left earlobe. Electrode impedances were monitored and maintained at $<5 \mathrm{kOhms}$. The EEG electrodes were connected to a standard amplifier system (Nihon Kohden). The signal was sampled at a $1 \mathrm{kHz}$ rate over a $0.5-500 \mathrm{~Hz}$ bandwidth. Respiratory-related potentials were evoked by mid-inspiratory occlusions [22]. To ensure immediate reproducibility of the signals, two separate averagings of 80 occluded breaths were systematically performed in each studied condition and the resulting traces superimposed. Control signals were obtained by averaging the same number of unoccluded breaths. The peak latencies of the first positive (P1), first negative (N1), second positive (P2) and second negative (N2) components of the RREP were measured from the onset of inspiration determined on the averaged mouth pressure trace according to DAVENPORT et al. [23]. The amplitude of the P1 component of the RREP was measured from baseline to peak. The amplitudes of the N1, P2 and N2 components were measured from peak to peak (P1-N1, N1-P2, and P2-N2, respectively).

Maximal static transdiaphragmatic pressure. Maximal static $P$ di ( $P$ di,max) was determined by asking the subjects to perform inspiratory efforts from functional residual capacity against the occluded airway, with visual feedback of the $P$ di signal. No instruction was given as to the type of manoeuvre to perform. The best of three attempts was retained.

Diaphragm challenge. To acutely modify diaphragm function, the subjects were asked to breathe against an inspiratory resistance while targeting an inspiratory $P$ di amounting to $60 \%$ of $P$ di,max with an inspiratory time of $\sim 50 \%$ of the ventilatory period, until task failure with strong verbal coaching. This protocol was chosen according to in-house observations showing that, in subjects without prior experience of inspiratory loading, it tends to produce changes in inspiratory muscle output predominantly due to a diaphragmatic contribution. In the authors experience, this result is achieved with no need to standardise the reference $P$ di,max manoeuvre and the pattern of muscle recruitment used during the loading procedure. The authors considered predominant diaphragm dysfunction present when a reduction in $P$ di,tw of $\geqslant 10 \%$ was observed, with a $P$ es,tw: $P$ ga,tw unchanged or increased [16].

Experimental conditions and sequence. The subjects were studied sitting (respiratory pressure measurements and fatigue 
protocol) or semireclined (respiratory related evoked potentials), abdomen unbound, on a lounge chair with the back, neck and head comfortably supported. They were instructed to relax but to keep their eyes open in order to avoid any risk of falling asleep or producing slow brain waves. During the experimental sequence, the subjects wore earplugs and headphones through which they listened to a quiet musical piece of their choice, in order to mask the auditory ambiance of the laboratory.

After completion of the experimental setup, baseline RREP were gathered and $P$ di, max was determined. Baseline diaphragm twitches were then collected, and the acute loading procedure was performed. Immediately after task failure, RREP were gathered again, after which diaphragm twitches were again obtained.

\section{Statistical analysis}

Data are described as mean \pm SD. Given the small size of the sample, and to avoid making assumptions on the distribution of the characteristics of the RREP, the analysis was conducted in a nonparametric way. The effects of inspiratory loading on twitch pressures were tested using the Wilcoxon test. Regarding the RREP, two steps were taken. First, when the number of observations of a given component in a given derivation differed before and after loading, a Chi-squared test with correction for small sample size was conducted to test the difference in a dichotomic manner. Secondly, the amplitude and latency of the various peaks in the $\mathrm{C} 3$ and $\mathrm{C} 4$ derivations before and after loading were compared using a nonparametric Kruskall-Wallis analysis of variance. Differences were considered significant when the probability of a type I error was $<5 \%$.

\section{Results}

\section{Diaphragm function}

Before loading, the $P$ di,max values in the nine subjects ranged $50-160 \mathrm{cmH}_{2} \mathrm{O}\left(88 \pm 35 \mathrm{cmH}_{2} \mathrm{O}\right)$, and the $P$ di, tw values were $27.25 \pm 5.52 \mathrm{cmH}_{2} \mathrm{O}$, within the normal range [18]. The time to task failure ranged $12-34 \mathrm{~min}$. In one subject, $P$ di,tw was unaffected by the procedure. This subject was therefore excluded from the analysis. In one additional subject, $P$ di,tw decreased by $>10 \%$ but the $P$ es, tw: $P$ ga,tw also decreased. This individual was also excluded from the analysis, which was therefore conducted in seven subjects who met the predefined criteria for a predominant diaphragmatic change ( $P$ es,tw: $P$ ga,tw increased in six cases, unchanged in one). The average $P$ di,tw after loading was $19.91 \pm 6.46 \mathrm{cmH}^{2} \mathrm{O}$, namely a $28 \pm 13 \%$ reduction (fig. 1) ( $\mathrm{p}=0.018$, power $85 \%$ for an alpha value of $0.05)$. The average reductions in $P$ es,tw and $P$ ga,tw were $14 \pm 8 \%(\mathrm{p}=0.009)$ and $51 \pm 25 \%(\mathrm{p}=0.002)$, respectively. The amplitudes of the electromyographical responses to phrenic stimulation were unaffected by the loading protocol (see Materials and methods).

\section{Respiratory-related evoked potentials}

Averaging the EEG signal corresponding to unoccluded breaths did not yield any potential. At baseline, RREP were identified in all subjects (table 1, table 2, and fig. 2). The P1, $\mathrm{N} 1$ and $\mathrm{P} 2$ components were present in either $\mathrm{C}_{3}$ or $\mathrm{C}_{4}$ in the seven cases, and bilaterally in four. The $\mathrm{N} 2$ component was present in either $\mathrm{C} 3$ or $\mathrm{C} 4$ in six cases, and again bilaterally in four. After acute loading, the P1 and N1 components were
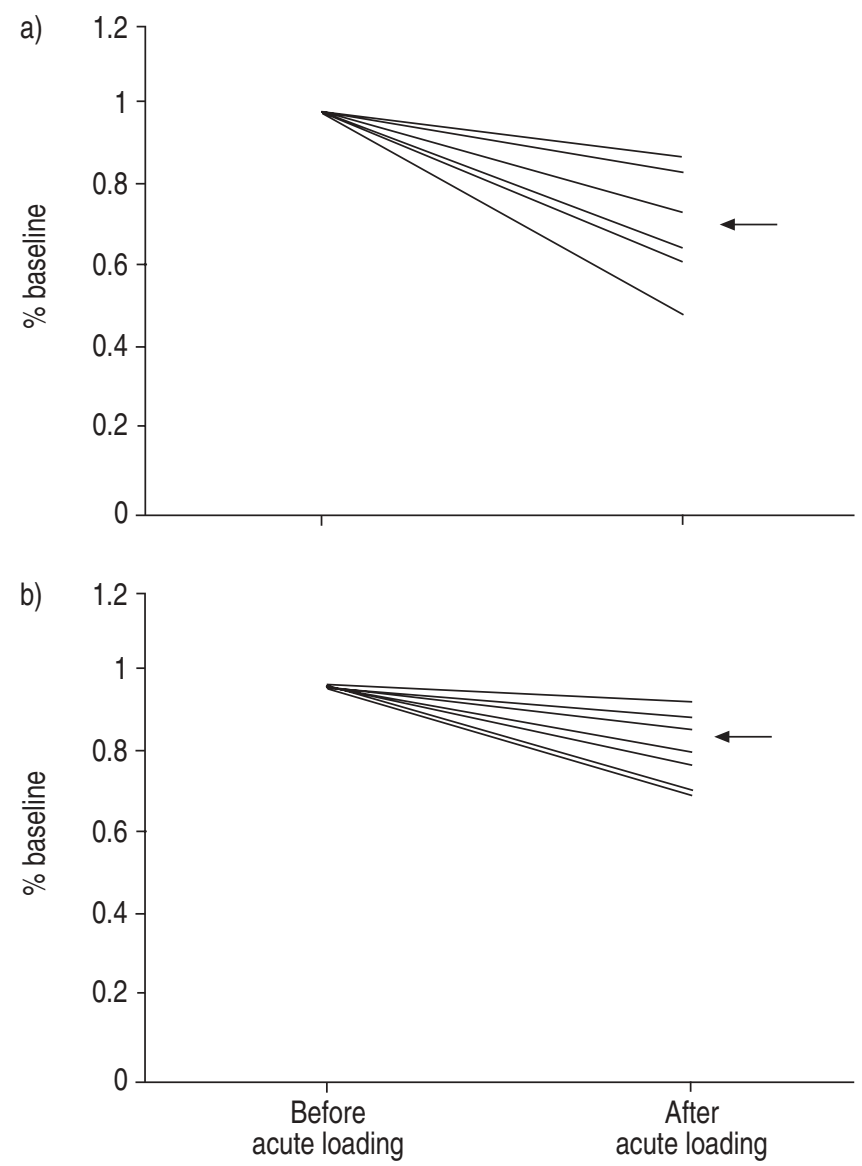

Fig. 1.- The effects of the acute inspiratory resistive loading protocol on the capacity of the diaphragm to produce a) transdiaphragmatic pressure and b) oesophageal pressure in the seven subjects retained for analysis. The "after" values are expressed in percentage of the "before" ones. The horizontal arrows indicate the mean of the "after" values.

also present in either $\mathrm{C} 3$ or $\mathrm{C} 4$ in the seven cases (always homolaterally as compared with baseline condition), and bilaterally in four. The $\mathrm{P} 2$ and the $\mathrm{N} 2$ components were present in either $\mathrm{C} 3$ or $\mathrm{C} 4$ in five cases, and bilaterally in four. There was no statistically significant difference regarding the number of occurrences of the components before and after fatigue (Chi-squared test, see methods). Among the seven subjects, the comparison of the amplitudes and latencies of the components before and after fatigue did not yield any significant difference (Kruskall-Wallis test, see Materials and methods).

\section{Discussion}

In this study, the characteristics of RREP were unaffected by an inspiratory resistive challenge imposed on healthy subjects to the point of task failure. This challenge resulted in an overt reduction of the transdiaphragmatic pressure produced in response to phrenic stimulation $(28 \pm 13 \%)$. The pattern of this reduction suggested a predominant impact on the diaphragm, rather than on extradiaphragmatic inspiratory muscles (although some degree of rib cage muscle dysfunction cannot be completely excluded in the absence of comparison of CMS with electrical phrenic nerve stimulation) [16].

Of note, the effects of loading on the response to phrenic stimulation were assessed after the collection of the RREP data, namely $\sim 15$ min after task failure. The observed lack of 
Table 1.-Latencies of the components of the respiratory-related evoked potentials (RREP) before and after inspiratory loading in the $\mathrm{C}_{\mathrm{Z}}-\mathrm{C} 3$ and $\mathrm{C}_{\mathrm{Z}}-\mathrm{C} 4$ scalp positions

\begin{tabular}{|c|c|c|c|c|c|c|c|c|}
\hline & \multicolumn{4}{|c|}{$\mathrm{C}_{\mathrm{Z}^{-}} \mathrm{C} 3$} & \multicolumn{4}{|c|}{$\mathrm{C}_{\mathrm{Z}^{-}} \mathrm{C} 4$} \\
\hline & Before fatigue & $\mathrm{n}^{\#}$ & After fatigue & $\mathrm{n}^{\#}$ & Before fatigue & $\mathrm{n}^{\#}$ & After fatigue & $\mathrm{n}^{\#}$ \\
\hline $\mathrm{P} 1$ & $38.30 \pm 5.96$ & 5 & $38.80 \pm 3.90$ & 5 & $38.00 \pm 3.32$ & 5 & $37.83 \pm 3.13$ & 6 \\
\hline $\mathrm{N} 1$ & $91.42 \pm 19.39$ & 6 & $96.25 \pm 18.08$ & 6 & $88.83 \pm 21.55$ & 6 & $94.30 \pm 16.36$ & 5 \\
\hline $\mathrm{P} 2$ & $144.83 \pm 23.15$ & 6 & $146.70 \pm 22.04$ & 5 & $129.67 \pm 18.00$ & 6 & $131.50 \pm 23.98$ & 4 \\
\hline N2 & $219.10 \pm 8.65$ & 5 & $209.10 \pm 9.79$ & 5 & $198.80 \pm 16.16$ & 5 & $191.00 \pm 18.50$ & 4 \\
\hline
\end{tabular}

Data are presented as mean \pm SD ms. The peak latencies of the first positive (P1) and first negative (N1) components of the RREP were present at least unilaterally in the seven cases, both before and after fatigue, in a homolateral manner. P2: peak latency of second positive component of the RREP; N2: peak latency of the second negative component of the RREP. ${ }^{\#}$ : indicates the number of occurrences of the different components in the seven subjects retained for analysis.

Table 2. - Amplitudes of the components of the respiratory-related evoked potentials (RREP) before and after inspiratory loading in the $\mathrm{C}_{Z}-\mathrm{C} 3$ and $\mathrm{C}_{Z}-\mathrm{C} 4$ scalp positions

\begin{tabular}{|c|c|c|c|c|c|c|c|c|}
\hline & \multicolumn{4}{|c|}{$\mathrm{C}_{\mathrm{Z}}-\mathrm{C} 3$} & \multicolumn{4}{|c|}{$\mathrm{C}_{\mathrm{Z}}-\mathrm{C4}$} \\
\hline & Before fatigue & $\mathrm{n}^{\#}$ & After fatigue & $\mathrm{n}^{\#}$ & Before fatigue & $\mathrm{n}^{\#}$ & After fatigue & $\mathrm{n}^{\#}$ \\
\hline $\mathrm{P} 1$ & $2.88 \pm 1.55$ & 5 & $2.80 \pm 0.84$ & 5 & $2.44 \pm 0.95$ & 5 & $2.25 \pm 0.94$ & 6 \\
\hline N1 & $-4.15 \pm 2.00$ & 6 & $-4.62 \pm 1.08$ & 6 & $-3.62 \pm 0.94$ & 6 & $-3.76 \pm 0.74$ & 5 \\
\hline $\mathrm{P} 2$ & $2.77 \pm 1.15$ & 6 & $2.94 \pm 0.66$ & 5 & $2.35 \pm 0.72$ & 6 & $1.90 \pm 0.67$ & 4 \\
\hline $\mathrm{N} 2$ & $-3.36 \pm 0.76$ & 5 & $-2.94 \pm 0.57$ & 5 & $-3.32 \pm 0.69$ & 5 & $-2.80 \pm 0.57$ & 4 \\
\hline
\end{tabular}

Data are presented as mean $\pm \mathrm{SD} \mu \mathrm{V}$. The peak latencies of the first positive (P1) and first negative (N1) components of the RREP were present at least unilaterally in the seven cases, both before and after fatigue, in a homolateral manner. P2: peak latency of second positive component of the RREP; N2: peak latency of the second negative component of the RREP. ${ }^{\#}$ : indicates the number of occurrences of the different components in the seven subjects retained for analysis.

change in these variables is thus not likely to be due to the effects of loading on inspiratory muscle function having waned between task failure and data collection.

RREP represent the respiratory counterpart of somatosensory evoked potentials. They correspond to the activity of cortical neurons in response to a mechanical inspiratory stimulus. Their first positive component, P1, is considered to reflect the cortical arrival of the afferent stimulus [24]. Their later components are considered to reflect the cortical processing of the afferent information. Identifying the determinants of the RREP is important, because this could provide a means to objectively quantify some aspects of respiratory sensations, and to assign objective numerical values to their changes after an intervention. In this view, the

a)
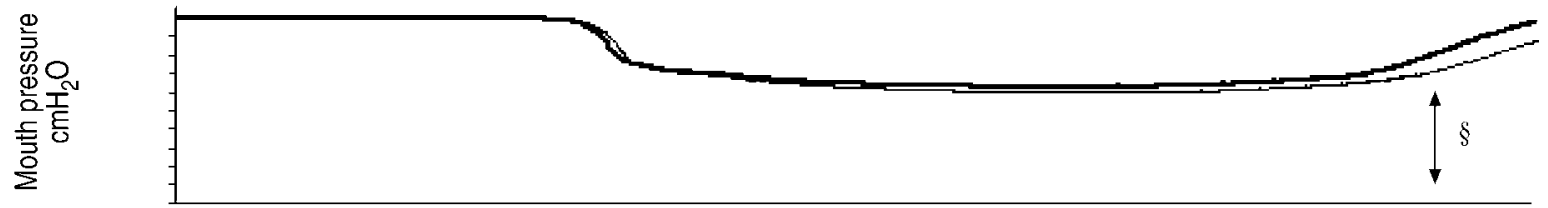

b)

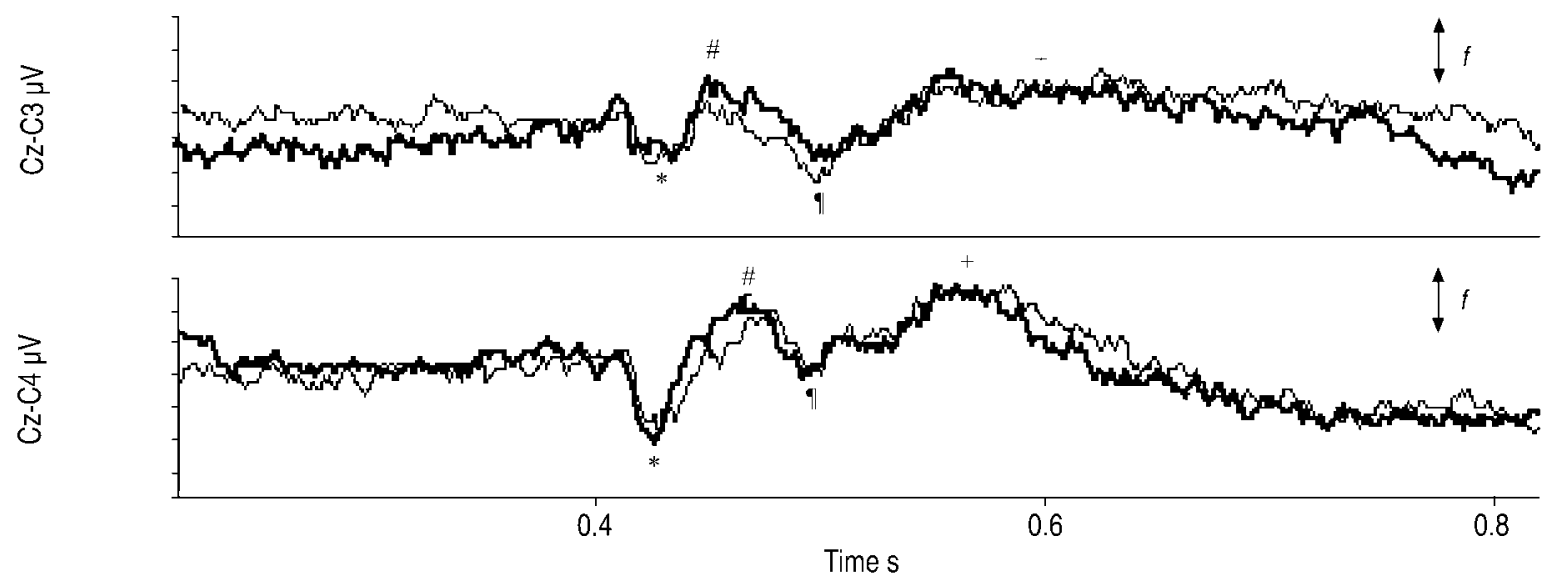

Fig. 2. - Example, in one subject, of respiratory-related evoked potentials including a) mouth pressure, b) $\mathrm{C}_{\mathrm{Z}}-\mathrm{C} 3$ and c) $\mathrm{C}_{\mathrm{Z}}-\mathrm{C} 4$, before $(-)$ and after (-) the acute loading protocol. Each trace in b) and c) corresponds to the ensemble averaging of two separate superimposable traces obtained from 80 mid-inspiratory occlusions. ${ }^{*}$ : first positive; ${ }^{\#}$ : first negative; ${ }^{\uparrow}$; second positive; ${ }^{+}$: second negative; ${ }^{\S}: 5 \mathrm{cmH}_{2} 0 ;{ }^{f}: 2 \mu \mathrm{V}$. 
P1 component appears particularly important. Its latency relates to the intensity of the inspiratory motor drive [23]. Its amplitude increases with the level of inspiratory effort developed to overcome a load [13]. The estimated magnitude of an inspiratory load relates to the amplitude of P1 $[12,13]$. In children having suffered near-fatal attacks of asthma (as opposed to "normal" asthmatic children and controls) [25], it has been shown that the RREP could lack the P1 peak, a finding that fits with the blunted perception of loads featured by severe asthmatics [2].

Many structures throughout the respiratory system can theoretically give rise to the afferent information underlying the RREP, but their location and nature are not precisely known. Data in lung transplant recipients suggest that vagally mediated afferents do not play a significant role [26]. Conversely, the role of the supraglottic receptors located in the upper airway is probably major [27], but currently available data leave open the possibility of downstream sources. These include the respiratory muscles. KNAFELC and DAVENPORT [13] indeed reported that the P1 peak amplitude increased in relation to the intensity of the inspiratory effort required to overcome a load. From another point of view, HUANG et al. [28] studied RREP in healthy subjects before and after inspiratory muscle training. In this study, inspiratory muscle strengthening, as ascertained by an increase in the subjects' ability to develop static inspiratory pressures, was associated with a significantly decreased motor drive to breathe. Globally, this study did not evidence statistically significant changes in the RREP. However, restricting the analysis to the somatosensory electrodes, the authors noted a significant reduction in the amplitude of the P1 component after training. It thus appears that acutely modifying the load imposed on inspiratory muscles [13] or chronically modifying their ability to develop inspiratory pressures [28] can change at least the characteristics of the P1 peak of the RREP. How the various inspiratory muscle groups (including the diaphragm) contribute to these changes is not known. Indeed, in the study by KNAFELC and DAVENPORT [13], the relationship between the amplitude of $\mathrm{P} 1$ and the inspiratory effort was present when the inspiratory effort was judged in terms of transdiaphragmatic pressure, but also in terms of the nonspecific oesophageal and mouth pressures. The training protocol used by HUANG et al. [28] did not put emphasis on any particular inspiratory muscle group. However, elucidating the respective contribution of afferents from the diaphragm and from other inspiratory muscles is of pathophysiological relevance because load-related dyspnoeic sensations differ when extradiaphragmatic inspiratory muscles, rather than the diaphragm, are predominantly involved in the load response $[14,15]$. This could influence the determinants of dyspnoea in diseases affecting inspiratory muscle groups differentially.

In subjects in the current study, modifying the ability of the diaphragm to produce inspiratory pressures did not result in significant changes in the characteristics of the various components of the RREP. In the hypothesis of an actual contribution of respiratory muscles to RREP, this does not exclude the diaphragm as a sensory information source, but suggests that the sensory processing arises from more than just the diaphragm and involves several inspiratory muscle groups. This result is in line with various types of data relating respiratory muscle function and respiratory sensations. Indeed, global inspiratory muscle dysfunction or fatigue, as induced by partial curarisation [6] and exhaustion following repeated maximal static inspiratory efforts [5] lead to the overestimation of inspiratory loads. Conversely, respiratory sensations seem preserved in situations where inspiratory muscle groups are altered separately. BRADLEY et al. [29], producing shifts in the high-to-low ratio of the frequency content of the diaphragmatic electromyographical signals through a loading protocol similar to that used in the current study, reported that the sense of inspiratory effort was independent of diaphragm fatigue. FITTING et al. [15] reported that the perceived magnitude of an inspiratory effort exerted against a load was independent of the emphasis put on the diaphragm. FRANKEL et al. [30] described the case of a tetraplegic patient with diaphragm atrophy but preserved inspiratory neck muscles in whom the perception and magnitude estimation of inspiratory loads were normal. Conversely, SINDERBY et al. [31] established a link between respiratory effort sensation and the frequency content of diaphragm electrical activity, but their loading protocol might however have led to global inspiratory fatigue.

In the above paragraphs, it was assumed that inspiratory muscle afferents contribute directly to the origin of the RREP, and therefore that inducing a predominant diaphragm dysfunction did not suffice to suppress this contribution. Alternatively, the generation of the airway occlusion-related evoked potentials measured in unloaded conditions could be independent from the strength of the inspiratory muscles. This hypothesis could be tested by studying the changes in the amplitude of $\mathrm{P} 1$ elicited by graded inspiratory resistances (instead of complete occlusions) before and after inspiratory fatigue. Of note, the results from the current study do not exclude modifications of the RREP as they would be measured during the loading challenge itself rather than after task failure. Indeed, diaphragm fatigue activates type IV afferents originating in respiratory muscles [32], which could account for the diaphragm-fatigue related changes in cortical potentials that have been described in the cat [11]. Finally, the contribution of respiratory muscle afferents to the RREP (a plausible possibility in view of intercostal and phrenic source of evoked potentials) [8-10] could be either inexistent or extremely small. In support of the latter idea, bypassing most of the upper airway with a laryngeal mask dramatically reduces the amplitude of the various components of the RREP [27].

In part, because of the relatively small size of the population studied, further studies are needed to clarify several issues raised from the current study. It would, in particular, be useful to correlate, in the same subjects and at the same time, the evolution of RREP and load detection and estimation after various types of interventions. However, the results support the notion, apparent in the literature, that an acute decrease in diaphragm function does not suffice to modify the sensory processing of inspiratory loads. Thus, there could be a redundancy between afferents from the diaphragm and afferents from other inspiratory muscles, which, considering respiratory sensations as an alarm system, could serve a safety purpose (this is similar to the notion that diaphragm fatigue does not prevent the inspiratory muscle system as a whole to adequately respond to hypercapnia [33]). This could have implications in the understanding of the mechanisms of dyspnoea during various types of respiratory disease.

Acknowledgements. The authors are grateful to M-H. Becquemin, L. Chalumeau-Lemoine, L. Mangin, M-S. Merino-Andreu, and J. Gonzalez for having accepted to serve as subjects in this study.

\section{References}

1. Manning HL, Schwartzstein RM. Pathophysiology of dyspnea. N Engl J Med 1995; 333: 1547-1553.

2. Kikuchi Y, Okabe S, Tamura G, et al. Chemosensitivity and 
perception of dyspnea in patients with a history of near-fatal asthma. N Engl J Med 1994; 330: 1329-1334.

3. Killian KJ, Campbell EJM. Dyspnea. In: Crystal R, West J, eds. The lung: scientific foundations. New York, Raven Press Ltd, 1991; p. 1433.

4. Kellerman BA, Martin AD, Davenport PW. Inspiratory strengthening effect on resistive load detection and magnitude estimation. Med Sci Sports Exerc 2000; 32: 1859-1867.

5. Gandevia SC, Killian KJ, Campbell EJ. The effect of respiratory muscle fatigue on respiratory sensations. Clin Sci 1981; 60: 463-466.

6. Campbell EJ, Gandevia SC, Killian KJ, Mahutte CK, Rigg JR. Changes in the perception of inspiratory resistive loads during partial curarization. J Physiol (Lond) 1980; 309: 93100 .

7. McCloskey DI. Muscular and cutaneous mechanisms in the estimation of the weights of grasped objects. Neuropsychologia 1974; 12: 513-520.

8. Gandevia SC, Macefield G. Projection of low-threshold afferents from human intercostal muscles to the cerebral cortex. Respir Physiol 1989; 77: 203-214.

9. Straus C, Zelter M, Derenne J-P, Pidoux B, Willer J, Similowski T. Putative projection of phrenic afferents to the limbic cortex in man studied with cerebral evoked potentials. J Appl Physiol 1997; 82: 480-490.

10. Zifko UA, Young BG, Remtulla H, Bolton CF. Somatosensory evoked potentials of the phrenic nerve. Muscle Nerve 1995; 18: 1487-1489.

11. Balzamo E, Lagier-Tessonnier F, Jammes Y. Fatigueinduced changes in diaphragmatic afferents and cortical activity in the cat. Respir Physiol 1992; 90: 213-226.

12. Knafelc M, Davenport PW. Relationship between resistive loads and P1 peak of respiratory-related evoked potential. J Appl Physiol 1997; 83: 918-926.

13. Knafelc M, Davenport PW. Relationship between magnitude estimation of resistive loads, inspiratory pressures, and the RREP P1 peak. J Appl Physiol 1999; 87: 516-522.

14. Ward ME, Eidelman D, Stubbing DG, Bellemare F, Macklem PT. Respiratory sensation and pattern of respiratory muscle activation during diaphragm fatigue. $J \mathrm{Appl}$ Physiol 1988; 65: 2181-2189.

15. Fitting JW, Chartrand DA, Bradley TD, Killian KJ, Grassino A. Effect of thoracoabdominal breathing patterns on inspiratory effort sensation. J Appl Physiol 1987; 62: $1665-1670$.

16. Similowski T, Straus C, Attali V, Duguet A, Derenne JP. Cervical magnetic stimulation as a method to discriminate between diaphragm and rib cage muscle fatigue. $J$ Appl Physiol 1998; 84: 1692-1700.

17. Verin E, Straus C, Demoule A, Mialon P, Derenne JP, Similowski T. Validation of improved recording site to measure phrenic conduction from surface electrodes in humans. J Appl Physiol 2002; 92: 967-974.

18. Green M, Road J, Sieck G, Similowski T. ATS/ERS Statement on respiratory muscle testing: Tests of respiratory muscle strength. Am J Respir Crit Care Med 2002; 166: 528547.
19. Similowski T, Fleury B, Launois S, Cathala HP, Bouche P, Derenne JP. Cervical magnetic stimulation: a new painless method for bilateral phrenic nerve stimulation in conscious humans. J Appl Physiol 1989; 67: 1311-1318.

20. Similowski T, Mehiri S, Attali V, Duguet A, Straus C, Derenne J-P. Comparison of magnetic and electrical phrenic nerve stimulation in assessment of phrenic nerve conduction time. J Appl Physiol 1997; 82: 1190-1199.

21. Kriss A. Recording technique. In: Halliday AM, ed. Evoked potentials in clinical testing. Edinburgh, Churchill Livingstone, 1993; pp. 1-56.

22. Revelette WR, Davenport PW. Effects of timing of inspiratory occlusion on cerebral evoked potentials in humans. J Appl Physiol 1990; 68: 282-288.

23. Davenport PW, Holt GA, Hill PM. The effect of increased inspiratory drive on the sensory activation of the cerebral cortex by inspiratory occlusion. In: Speck DF, Dekin MS, Revelette WR, Frazier DT, eds. Respiratory control: central and peripheral mechanisms. Lexington, USA, University Press of Kentucky, 1992; pp. 216-221.

24. Davenport PW, Friedman WA, Thompson FJ, Franzen O. Respiratory-related cortical potentials evoked by inspiratory occlusion in humans. J Appl Physiol 1986; 60: 1843-1848.

25. Davenport PW, Cruz M, Stecenko AA, Kifle Y. Respiratoryrelated evoked potentials in children with life-threatening asthma. Am J Respir Crit Care Med 2000; 161: 1830-1835.

26. Zhao W, Martin AD, Davenport PW. Respiratory-related evoked potentials elicited by inspiratory occlusions in double-lung transplant recipients. J Appl Physiol 2002; 93: 894-902.

27. Daubenspeck JA, Manning HL, Akay M. Contribution of supraglottal mechanoreceptor afferents to respiratoryrelated evoked potentials in humans. J Appl Physiol 2000; 88: 291-299.

28. Huang $\mathrm{CH}$, Martin AD, Davenport PW. Effect of inspiratory muscle strength training on inspiratory motor drive and RREP early peak components. J Appl Physiol 2003; 94: 462.

29. Bradley TD, Chartrand DA, Fitting JW, Killian KJ, Grassino A. The relation of inspiratory effort sensation to fatiguing patterns of the diaphragm. Am Rev Respir Dis 1986; 134: 1119-1124

30. Frankel HL, Guz A, Noble M. Respiratory sensations in patients with cervical cord transection. Paraplegia 1971; 9: $132-136$

31. Sinderby C, Spahija J, Beck J. Changes in respiratory effort sensation over time are linked to the frequency content of diaphragm electrical activity. Am J Respir Crit Care Med 2001; 163: 905

32. Hill JM. Discharge of group IV phrenic afferent fibers increases during diaphragmatic fatigue. Brain Res 2000; 856: $240-244$

33. Yan S, Lichros I, Zakynthinos S, Macklem PT. Effect of diaphragmatic fatigue on control of respiratory muscles and ventilation during CO2 rebreathing. J Appl Physiol 1993; 75: $1364-1370$. 